\title{
The Effects of Sugar Cane Policosanol on the LDL, HDL, Triglyceride and Total Cholesterol Levels of Dyslipidemic Patients: A Meta-Analysis
}

\author{
Miguel Paolo Patrocinio, Nathaniel Paragas, John Angelo Perez, Diana-Lynn S Que*, Kevin Quiogue, Matthew Rommel Reyes, \\ Maria Carmela Rigunay, Iris Katarina Sanchez, Pia Angelyn Sandoval, Marissa Kathrina Santos, Mae Fatima So, Eunice Joy \\ Soliveres, Bernhard Arris Tan
}

Faculty of Clinical Research, St. Luke’s Medical Center-Quezon City, Philippines

*Corresponding author: Diana-Lynn S Que, Neurology Resident, Institute of Neurosciences St. Luke’s Medical Center-Quezon City, Philippines. Email: dianalynne88@gmail.com

Citation: Patrocinio MP, Paragas N, Perez JA, Que DLS, Quiogue K, et al. (2017) The Effects of Sugar Cane Policosanol on the LDL, HDL, Triglyceride and Total Cholesterol Levels of Dyslipidemic Patients: A Meta-Analysis. Anesth Med Pract J: AMPJ117. DOI: 10.29011/AMPJ-117. 100017

Received Date: 30 October, 2017; Accepted Date: 10 November, 2017; Published Date: 20 November, 2017

\begin{abstract}
Background: Policosanol, a plant-wax extract composed of a mixture of eight high molecular mass aliphathic alcohol, is commonly used as a supplement to reduce total cholesterol \& LDL cholesterol in patients with dyslipidemia. Despite its widespread use, studies showed conflicting conclusions regarding its efficacy.

Objective: The objective of this research is to consolidate the available research findings regarding the efficacy and benefits of policosanol and to compare the effects of policosanol versus placebo on the lipid profile (total cholesterol, LDL-C, HDL-C, and triglycerides) of dyslipidemic patients.

Materials and Methods: A literature search was done using PUBMED database using "Policosanol (MESH or free text) AND dyslipidemia (MESH or free text)" limited to human subjects, male and female, clinical trials, and randomized controlled trials. Free text using the same keywords using Google Scholar search engine was also done. Three independent reviewers conducted validity assessment for each included study. Review Manager was used to construct Forest and funnel plots for each outcome. Subgroup analyses were also performed to the following variables: policosanol dose (10 mg and $20 \mathrm{mg})$, duration ( 8 weeks and 12 weeks) and patient age (those between 40-80 y/o).
\end{abstract}

Results: A total of 14 studies were retrieved from the electronic search, four were excluded because of not being able to fulfill the inclusion criteria. All studies were randomized controlled trials, double blinded studies, and placebo controlled. One study made use of double-dummy trial. A total of 875 participants were given policosanol, irrespective of dosage and the duration of treatment while 878 received placebo treatment. Overall percent change in total cholesterol favored policosanol (-7.24[-11.99, -3.29]) with a significant difference $(\mathrm{p}=0.0003)$. A significant difference was likewise seen in LDL-C ( $\mathrm{p}=0.001)$ also favoring policosanol (-8.67[-13.99. -3.34]), as well as in HDL-C $(9.94[3.32,15.65], \mathrm{p}=0.003)$. However, the studies were very much heterogeneous $\left(\mathrm{p}<0,00001, \mathrm{I}^{2}=100 \%\right)$. No significant difference $(\mathrm{p}=0.30)$ was seen in terms of triacyl glycerides. On subgroup analysis using $10 \mathrm{mg}$ of policosanol versus placebo for 12 weeks, a significant difference $(\mathrm{p}<0.00001)$ was found favoring policosanol in terms of total cholesterol (-17.93 [-19.85, -16.00]), LDL-C (-22.08 [-25.91, -18.26]) and HDL-C (23.05 [20.28, 25.82]). However, on subgroup analysis of studies that used $20 \mathrm{mg}$ of policosanol versus placebo for 12 weeks, no significant difference was seen in all parameters.

Conclusion: In conclusion, policosanol was found to significantly improve the lipid profile parameters specifically total cholesterol, LDL-C, and HDL-C but not TAGs in dyslipidemia patients at dose of 10mg for 8 weeks. Side effects were very minimal or even none at all, and thus policosanol is generally safe and well tolerated. The heterogeneity of the studies may reflect the inconsistent and highly variable state of current literature and may be attributed to the differences in methodologies and demographic parameters of the subjects. 


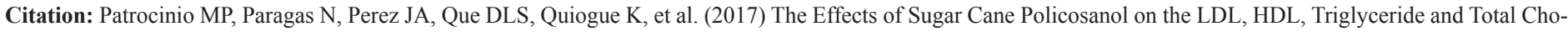
lesterol Levels of Dyslipidemic Patients: A Meta-Analysis. Anesth Med Pract J: AMPJ-117. DOI: 10.29011/AMPJ-117. 100017

Keywords: Dyslipidemia; hypercholesterolemia; Lipid; Policosanol

\section{Introduction}

Cardiovascular Disease (CVD) remains the number one cause of death globally, with 17.3 million people dying from CVD in 2008, yet projected to increase to 23.3 million by 2030 (World Health Organization, 2013) [1]. Low- and middle-income countries are mostly affected, with over $80 \%$ of CVD deaths occurring in these countries including the Philippines. The risk factors that lead to CVD include hyperlipidemia, an unhealthy diet, obesity, physical inactivity, hypertension and diabetes (WHO, 2013) [1].

Hyperlipidemia, clinically measured as the presence of LowDensity Lipoproteins (LDL) greater than or equal to $160 \mathrm{mg} / \mathrm{dL}$ after 12 hours of fasting, is a heterogenous group of disorders including hypercholesterolemia and hypertriglyceridemia. The former is defined as having total blood cholesterol levels greater than or equal to $240 \mathrm{mg} / \mathrm{dL}$ after 12 hours of fasting, while the latter is characterized by excess of lipids in the bloodstream. Cholesterol, cholesterol esters, phospholipids and triglycerides are all insoluble in the bloodstream and therefore have to be transported bound to lipoproteins.

Lipoproteins may be classified according to their protein component densities. In general, most cholesterol is carried in low-density lipoproteins and High-Density Lipoproteins (HDL), whereas most triglycerides are transported in chylomicrons by Very Low-Density Lipoproteins (VLDL). High levels of LDL are associated with an increased risk of atherosclerosis and coronary heart disease, while high levels of HDL are cardioprotective. Factors that may contribute to elevated levels of LDL include diet, obesity, inherited diseases, and other endocrinologic problems such as diabetes (Clinical Practice Guidelines).

In prevalence studies done in 2008 for Filipino adults, around $41.8 \%$ of all Filipino adults have increased cholesterol levels, while $10.2 \%$ have very high total cholesterol and $11.8 \%$ have very high LDL levels. This is an increase in prevalence from $8.5 \%$ and $11.7 \%$, respectively in 2003. A temporal profile of this prevalence shows that cholesterol levels have been increasing steadily since 1992 and this trend is expected to continue in the following years. Since dyslipidemia and hypercholesterolemia are independent risk factors for developing CVD, proper control is warranted, especially in patients with other CVD risk factors and hypercholesterolemia (Clinical Practice Guidelines).

At present, the primary treatment for patients with hypercholesterolemia centers around lifestyle changes such as smoking cessation, limiting alcohol consumption, increasing physical activity, and maintaining a healthy weight. These prevent other CVD comorbidities from occurring. A diet with low-cholesterol food, restricted saturated fats, and minimal trans-fat is likewise recom- mended. According to the Philippine Heart Association, patients with at least 3 risk factors for developing CVD and borderline levels of LDL and total cholesterol levels are already advised to take drug therapy. If lifestyle modifications fail to reduce CVD risk factors or lower cholesterol levels, then drugs such as statins and fibrates may be used. Statins inhibit the enzyme HMG-CoA reductase, an enzyme that plays a central role in the production of up to $70 \%$ of the body's cholesterol in the liver. On the other hand, fibrates are adjuncts to statins that work via various modes, with the end result being reduction of LDL, triglycerides and increasing HDL levels (Clinical Practice Guidelines).

Recently, increasing resistance to statin therapy has been documented and some therapeutic regimens are not recommended for those without additional risk factors for CVD. With these, food supplements claiming to help reduce cholesterol have been emerging in the market. One of these is policosanol, a natural product composed of a mixture of eight high molecular mass aliphatic alcohols extracted from plant waxes obtained most commonly from sugarcane [2]. It is commonly used as a supplement to reduce total cholesterol \& LDL cholesterol since the 1990s. Proposed mechanisms of action of policosanol include modulation of HMG-CoA reductase [3] and inhibition of bile acid absorption. Despite its widespread use, published studies have come to conflicting conclusions regarding its efficacy. A number of studies showed that policosanol has significant benefits of lowering LDL, total cholesterol and triglycerides [4], and increasing HDL [5-8]. However, there are also some studies that contradicted such benefits [9-15]. A study by Castano, et al. (2003) [16] showed that even though policosanol had significant effects on the lipid profile levels when compared to a statin (Atorvastatin ${ }^{\circledR}$ ), it was less effective in reducing the LDL and TC. However, it was shown in the same study that policosanol was able to cause a significant increase in serum HDL not found with Atorvastatin ${ }^{\circledR}$.

A meta-analysis has been done to compare the benefits of policosanol with those of plant sterols and stanols [17]. This showed that policosanol was more effective than plant sterols and stanols in lowering LDL levels. A significant reduction in LDL and triglycerides were found, while an increase in HDL was also observed. Another meta-analysis done locally compared the effects of sugar cane policosanol with placebo or non-treatment with total cholesterol and LDL as outcome measures [18]. While the meta-analysis was relatively recent and was generally valid, it only included 3 articles in the analysis and did not look into measures of HDL and triglycerides. HDL and triglyceride levels are as important as LDL and total cholesterol levels in assessing risk for CVD. In fact, a study by Sigdel showed that HDL and triglycerides are even better discriminating parameters than LDL for myocardial infarction. In addition, fasting blood sample is not required for HDL determination, unlike LDL which consequently may be incorrectly calculated in the presence of postprandial hypertriglyc- 


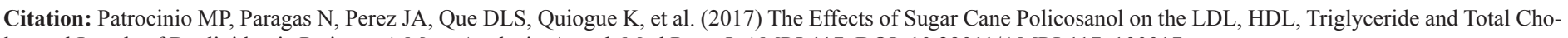
lesterol Levels of Dyslipidemic Patients: A Meta-Analysis. Anesth Med Pract J: AMPJ-117. DOI: 10.29011/AMPJ-117. 100017

eridemia. Furthermore, according to guidelines from the American Heart Association (AHA), HDL and triglycerides, but not LDL or total cholesterol comprise two of the five conditions to fulfill the criteria for metabolic syndrome, which is considered another risk factor for coronary heart disease, as well as for diabetes, fatty liver, and several cancers [19].

\section{Research Question and Objectives}

The current study then asks the question, what are the effects of sugar cane policosanol on the percent changes in the lipid profiles of dyslipidemic patients? The objective then of this research is to consolidate the available research findings regarding the efficacy and benefits of policosanol and to compare their effects to placebo on the lipid profile of dyslipidemic patients.

\section{Methodology}

\section{Inclusion Criteria and Exclusion Criteria}

The studies included were randomized controlled trials with dyslipidemic patients aged 18 years old to 80 years old of either gender. Dyslipidemia was defined as LDL $>130 \mathrm{mg} / \mathrm{dL}$, total cholesterol $>130 \mathrm{mg} / \mathrm{dL}$ and fasting triglycerides $<240 \mathrm{mg} / \mathrm{dL}$. The study included trials with treatment arms of 10-20mg of sugar policosanol for 8-12 weeks without any complimentary medications and excluded modified policosanol variants. There should also have been a concomitant control arm of placebo with the same duration. There was no restriction on frequency of treatment. The outcome measures were the percent changes in the components of the lipid profile: serum LDL, HDL, Triglycerides (TAGs), and Total Cholesterol (TC).

\section{Details of Literature Search}

A literature search was performed using the PUBMED database using "Policosanol (MESH or free text) AND dyslipidemia (MESH or free text)" limited to human subjects, male and female, clinical trials, and randomized controlled trials. Overall, 29 articles were recovered but among these only 12 full-text articles were retrieved. Free text using the same keywords using Google Scholar search engine was also done and was able to yield two additional articles. Among the 14 articles, two were excluded because one was a meta-analysis [17] and the other used wheat germ policosanol as the intervention instead of sugar policosanol. Two other studies by Kassis, et al. in 2006 and 2009 [13,14] were likewise excluded from the meta-analysis because the duration of intervention was only 4 weeks. A total of 10 studies were used for the final meta-analysis.

\section{Quality Assessment Criteria and Data Collection}

Three independent reviewers conducted validity assessment for each included study. They were assessed and included if they met the previously set inclusion criteria. Careful appraisal was done and quality assessment ratings given for methodology, adequacy of allocation concealment, blinding and follow-up rates. Disagreements were resolved by a third reviewer. As seen in (Table 1), majority of the studies had low risk of bias based on the critical appraisal by the reviewers. However, four studies were appraised as having high risk of bias primarily because they lacked intentionto-treat analysis despite having drop-outs. It is possible that the drop-outs had results that favored control (worst case scenario) and hence it is important to still include them in the final analysis.

\begin{tabular}{|c|c|c|c|c|c|c|c|}
\hline \multicolumn{4}{|c|}{ Primary } & \multicolumn{4}{|c|}{ Secondary } \\
\hline $\begin{array}{c}\text { Study (Author, } \\
\text { Year) }\end{array}$ & Randomization & Follow- up & ITT & Blinding & $\begin{array}{c}\text { Seminar Base- } \\
\text { line Character- } \\
\text { istics }\end{array}$ & $\begin{array}{c}\text { Group Treated } \\
\text { Equally }\end{array}$ & Rating \\
\hline $\begin{array}{c}\text { Torres, etal (1995) } \\
{[5]}\end{array}$ & Adequate & Not Adequate & Not Done & Double- Blind & Yes & Yes & $\mathrm{C}$ \\
\hline Mas, etal. (1999) [6] & Adequate & Adequate & Not Done & Double- Blind & Yes & Yes & $\mathrm{C}$ \\
\hline $\begin{array}{c}\text { Castano, etal. (2000) } \\
{[7]}\end{array}$ & Adequate & Adequate & Not Done & Double- Blind & Yes & Yes & $\mathrm{C}$ \\
\hline $\begin{array}{c}\text { Castano, etal. (2002) } \\
{[16]}\end{array}$ & Adequate & Adequate & Done & Double- Blind & Yes & Yes & A \\
\hline $\begin{array}{l}\text { Berthold, etal. } \\
(2006)[10]\end{array}$ & Adequate & Adequate & Done & Double- Blind & Yes & Yes & A \\
\hline $\begin{array}{c}\text { Cubeddu, etal. } \\
\text { (2006) [11] }\end{array}$ & Adequate & Adequate & Not specified & Double- Blind & Yes & Yes & $\mathrm{C}$ \\
\hline $\begin{array}{l}\text { Francini-Pesenti, } \\
\text { etal. (2006) [15] }\end{array}$ & Adequate & Adequate & Done & Double- Blind & Yes & Yes & A \\
\hline Dulin (2006) [12] & Adequate & Adequate & Done & Double- Blind & Yes & Yes & A \\
\hline
\end{tabular}




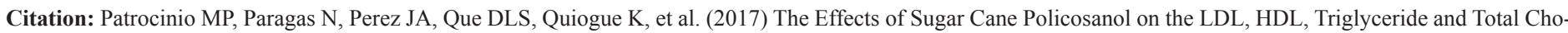
lesterol Levels of Dyslipidemic Patients: A Meta-Analysis. Anesth Med Pract J: AMPJ-117. DOI: 10.29011/AMPJ-117. 100017

\begin{tabular}{|c|c|c|c|c|c|c|c|}
\hline $\begin{array}{c}\text { Greyling, etal. } \\
(2006)[12]\end{array}$ & Adequate & Adequate & Done & Double- Blind & Yes & Yes \\
\hline $\begin{array}{c}\text { Illnait, etal. (2013) } \\
{[8]}\end{array}$ & Adequate & Adequate & Done & Double- Blind & Yes & Yes \\
\hline
\end{tabular}

Table 1: Summary of Critical Appraisal.

The percent change of each outcome and their standard deviations, if available, were obtained and encoded using Review Manager 5.2.7.

\section{Statistical Analysis}

Only 2 of the 10 included studies reported the standard deviations of their outcomes' percent change. In order to complete the meta-analysis, the researchers imputed the missing standard deviations by substituting them with the lowest standard deviation available in each analysis. The researchers initially attempted to impute standard deviations using correlation coefficients based on Berthold et al. 2006 [10] as prescribed by the Cochrane Collaboration, but found the results unacceptable due to unrealistically precise standard deviations.

Forest and funnel plots were constructed for each outcome using Review Manager. Statistically significant heterogeneity was almost universally encountered hence random effects models with a confidence interval of 95\% were used. Subgroup analyses were also performed according to the following variables: policosanol dose (10mg and $20 \mathrm{mg}$ ), duration ( 8 weeks and 12 weeks), their combinations, patient age (those $>18$ years old and those between $40-80$ years old) and their comorbidities.

\section{Results}

\section{Description of the Studies}

A total of fifteen studies were retrieved from the electronic search. Four were excluded because of not being able to fulfill the inclusion criteria. The characteristics of each study are shown in (Table 2). All the studies were randomized controlled trials, double blinded studies, and placebo controlled. One study (Cubeddu, 2006) made use of double-dummy trial.

\begin{tabular}{|c|c|c|c|c|c|}
\hline Study & Intervention, Dosage & Control & Age of Participants & No. of Participants & $\begin{array}{l}\text { Duration of follow- } \\
\text { up (weeks) }\end{array}$ \\
\hline Torres, etal. (1995) [5] & Policosanol, 10mg & Placebo & $55-57 \pm 10$ & 29 & 12 \\
\hline Mas, etal. (1999) [6] & Policosanol, 10mg & Placebo & $25-75$ & 437 & 12 \\
\hline $\begin{array}{c}\text { Castano etal. (2000) } \\
\text { [7] }\end{array}$ & Policosanol, 10mg & Placebo & $45-75$ & 244 & 12 \\
\hline $\begin{array}{c}\text { Berthold, etal. (2006) } \\
{[10]}\end{array}$ & $\begin{array}{l}\text { Policosanol, } 10 \mathrm{mg} \\
\text { and } 20 \mathrm{mg}\end{array}$ & Placebo & $18-80$ & 216 & 12 \\
\hline Illnait, etal. (2013) [8] & Policosanol, 10mg & Placebo & 57 & 90 & 12 \\
\hline $\begin{array}{c}\text { Dulin, etal. (2006) } \\
{[12]}\end{array}$ & Policosanol, 20mg & Placebo & $>18$ & 40 & 8 \\
\hline $\begin{array}{c}\text { Francini-Pesenti, etal. } \\
(2008)[15]\end{array}$ & Policosanol, 20mg & Placebo & $20-65$ & 68 & 8 \\
\hline $\begin{array}{c}\text { Greyling, etal (2006) } \\
{[9]}\end{array}$ & Policosanol, 20mg & Placebo & $>18$ & 19 & 12 \\
\hline $\begin{array}{c}\text { Cubeddu, etal. (2006) } \\
{[11]}\end{array}$ & Policosanol, 20mg & Placebo & $>20$ & 99 & 12 \\
\hline $\begin{array}{c}\text { Castano, etal. (2002) } \\
\text { [16] }\end{array}$ & Policosanol, 5mg & Placebo & $62-82$ & 589 & 12 \\
\hline
\end{tabular}

Table 2: Characteristics of each study. 


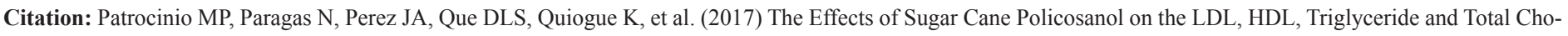
lesterol Levels of Dyslipidemic Patients: A Meta-Analysis. Anesth Med Pract J: AMPJ-117. DOI: 10.29011/AMPJ-117. 100017

Subjects included in the studies were hypercholesterolemic patients 18 years old to 80 years old. Five studies $[5,7,8,12,16]$ were on the elderly and postmenopausal population 40 to 80 years of age while 2 studies $[5,16]$ involved subjects with comorbid conditions, respectively non-insulin dependent diabetes mellitus and hypertension without any history of coronary heart disease or cerebrovascular disease. The study by Mas in 1999 specifically involved subjects who had other coronary risk factors; while the study by Greyling in 2006 [9] also included subjects with heterozygous familial hypercholesterolemia. Majority of the studies excluded subjects with active renal disease and severe liver disease or other severe diseases, smokers, diagnosed neoplastic diseases, severe hypertension with diastolic pressure of $120 \mathrm{mmHg}$, pregnant or nursing women, uncontrolled diabetes, history of cardiovascular diseases including myocardial infarction, stroke, or coronary surgery three months prior to the study, those taking any other lipid-lowering medication different from the study medication; those with triglyceride serum levels above $300 \mathrm{mg} / \mathrm{dL}$ using steroidal drugs, thyroid hormones and estroprogestinic preparations in the past six months.
All the studies used placebo as control. Except for Castano 2002 [16], they all measured the short-term effects of policosanol on lipid profile-12 weeks of $10 \mathrm{mg}$ policosanol vs. placebo $[5-8,10]$ 8 weeks of $20 \mathrm{mg}$ policosanol vs. placebo $[12,15]$, and 12 weeks of 20mg policosanol vs. placebo [9-11]. The study by Castano in 2002 [16] measured the long-term effects within 12 months of treatment with policosanol.

\section{Effects of Intervention}

A total of 875 participants were given policosanol, irrespective of the dose and duration of treatment while 878 participants received placebo treatment. Overall percent change in total cholesterol favored policosanol $(-7.24[-11.19,-3.29])$ with a significant difference $(\mathrm{p}=0.0003)$. A significant difference was likewise seen in LDL $(\mathrm{p}=0.001)$ also favoring policosanol $(-8.67$ [-13.99, -3.34]), as well as in HDL $(9.49$ [3.32, 15.65], $\mathrm{p}=0.003)$. However, the studies were very heterogenous ( $p<0.00001, \mathrm{I} 2=100 \%)$. No statistically significant results $(\mathrm{p}=0.30)$ were found for triglycerides (See Figures Below).

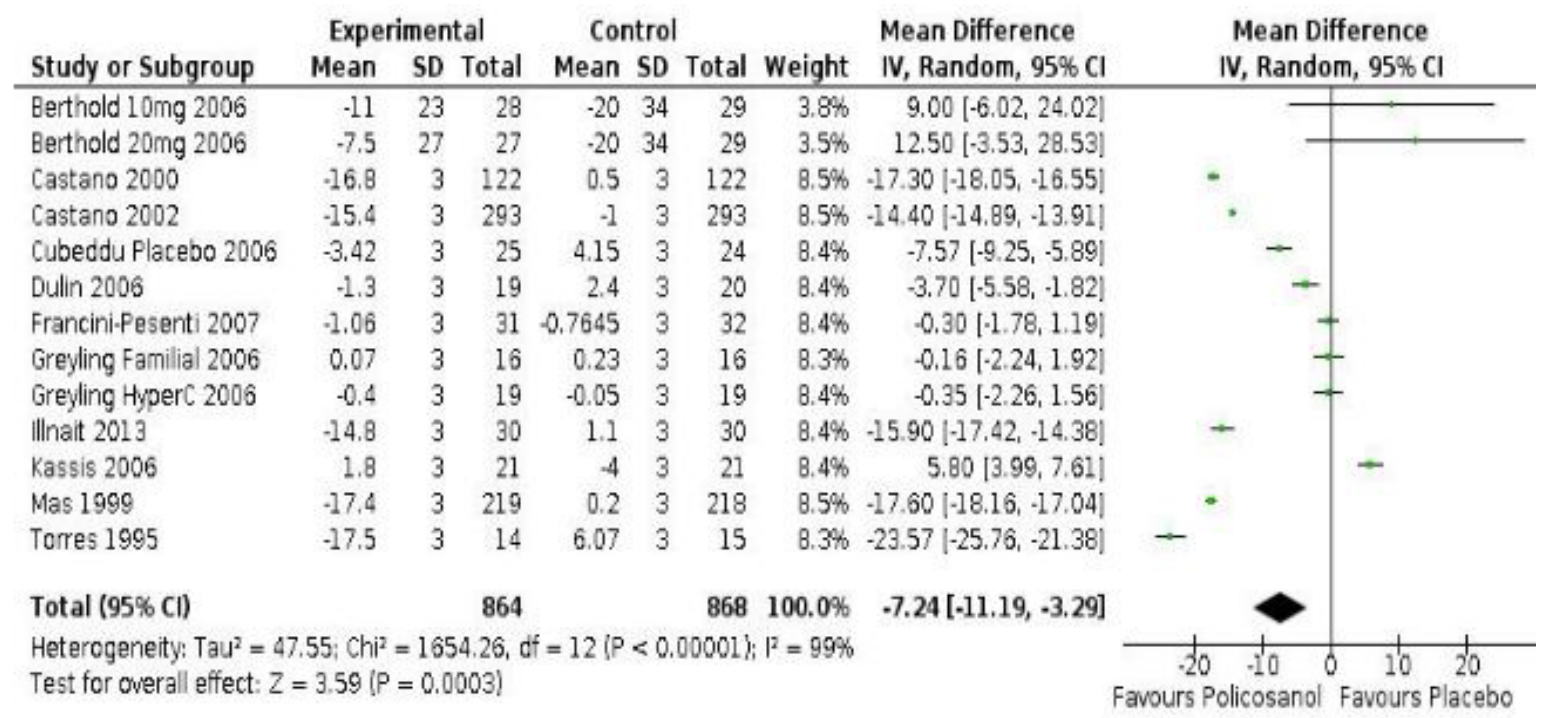

Figure 1: Policosanol vs. Placebo - Effect on total cholesterol. 
Citation: Patrocinio MP, Paragas N, Perez JA, Que DLS, Quiogue K, et al. (2017) The Effects of Sugar Cane Policosanol on the LDL, HDL, Triglyceride and Total Cholesterol Levels of Dyslipidemic Patients: A Meta-Analysis. Anesth Med Pract J: AMPJ-117. DOI: 10.29011/AMPJ-117. 100017

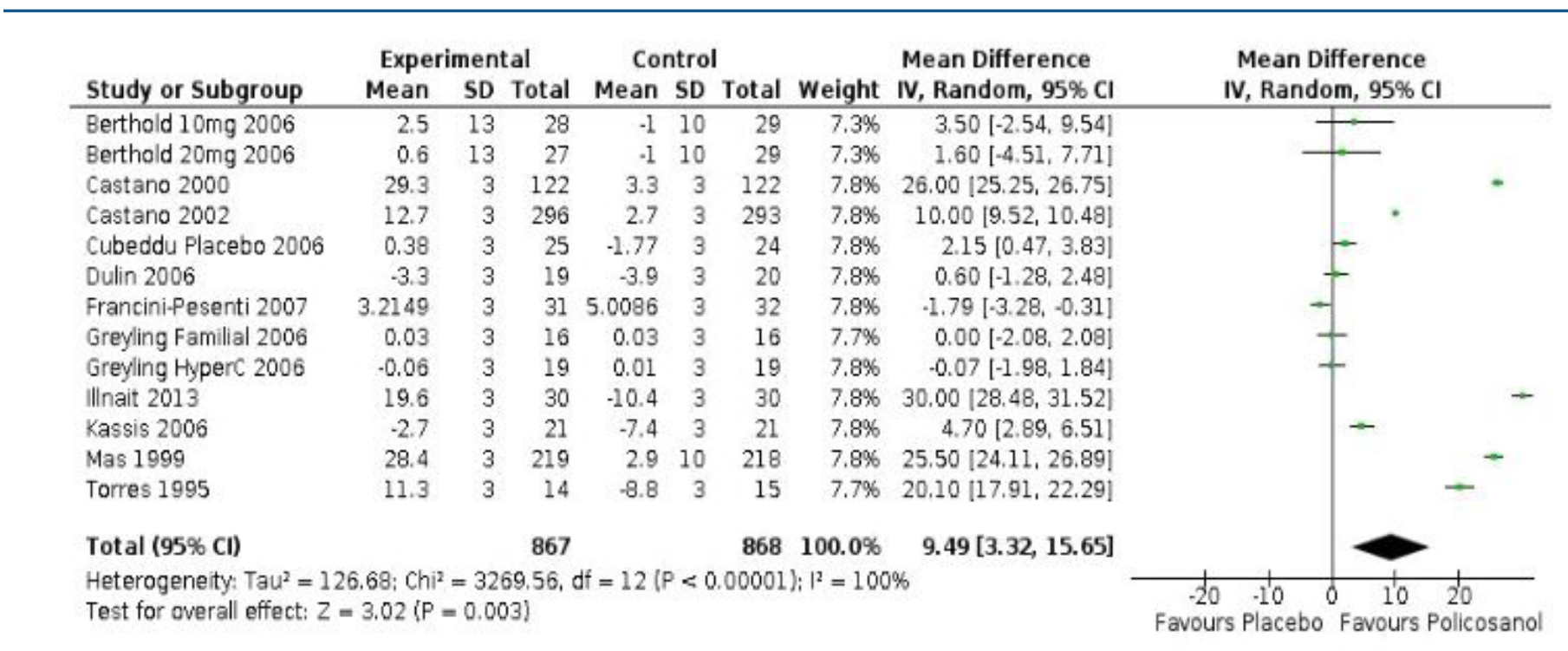

Figure 2: Policosanol vs. Placebo - Effect on LDLs.

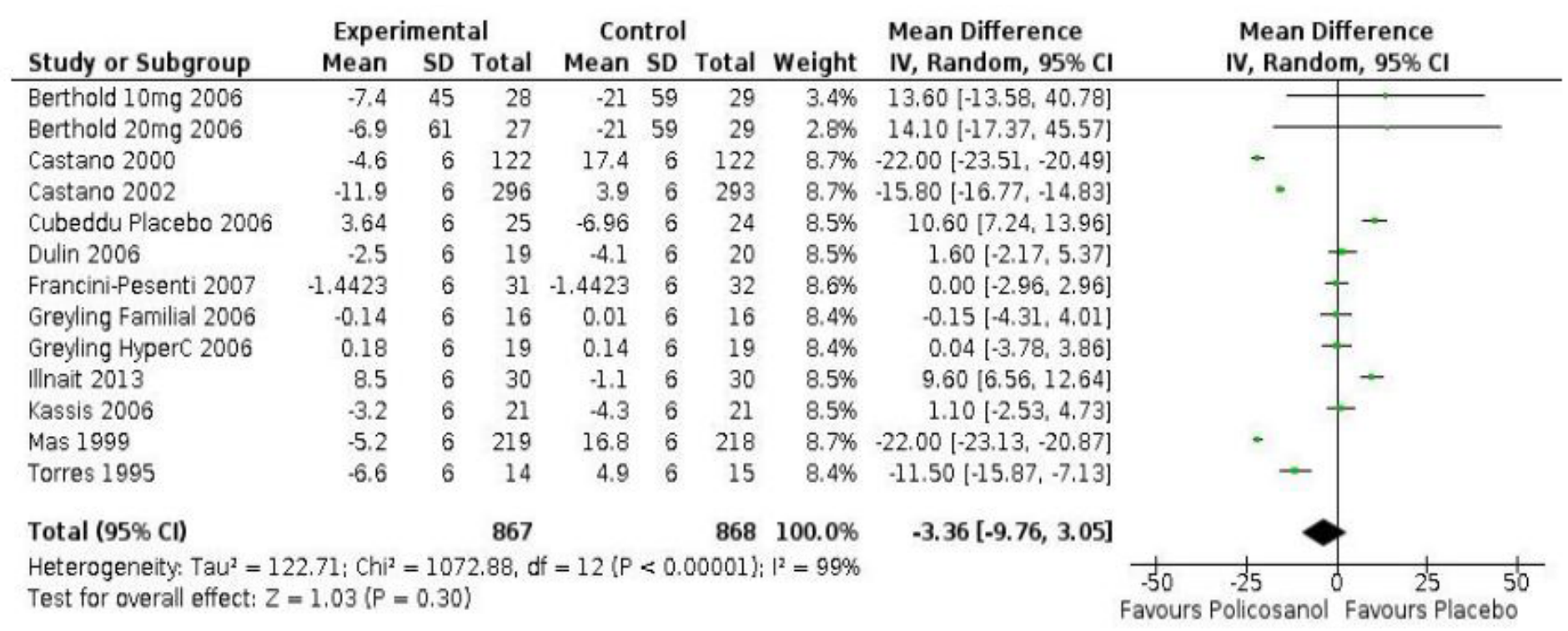

Figure 3: Policosanol vs. Placebo - Effect on HDLs

Funnel plots were done and in general, they were asymmetric (see figures below). 
Citation: Patrocinio MP, Paragas N, Perez JA, Que DLS, Quiogue K, et al. (2017) The Effects of Sugar Cane Policosanol on the LDL, HDL, Triglyceride and Total Cholesterol Levels of Dyslipidemic Patients: A Meta-Analysis. Anesth Med Pract J: AMPJ-117. DOI: 10.29011/AMPJ-117. 100017

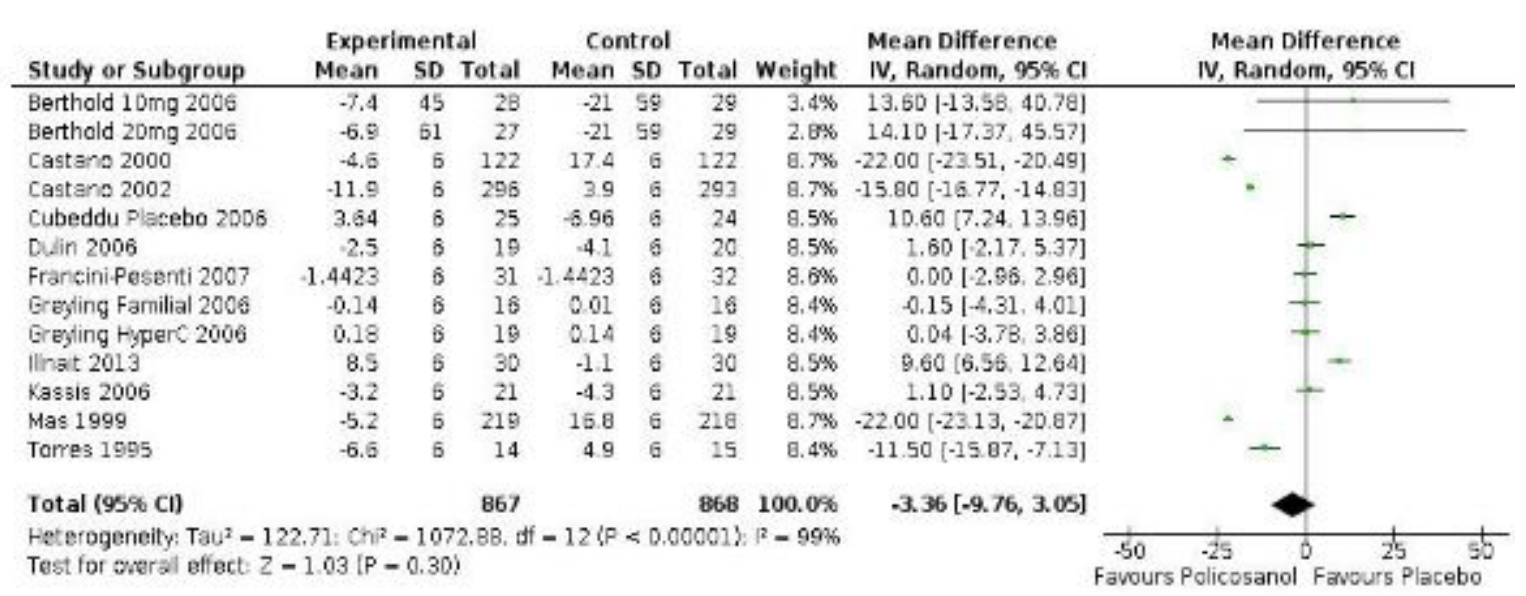

Figure 4: Policosanol vs. Placebo - Effect on Triacyl glycerides.
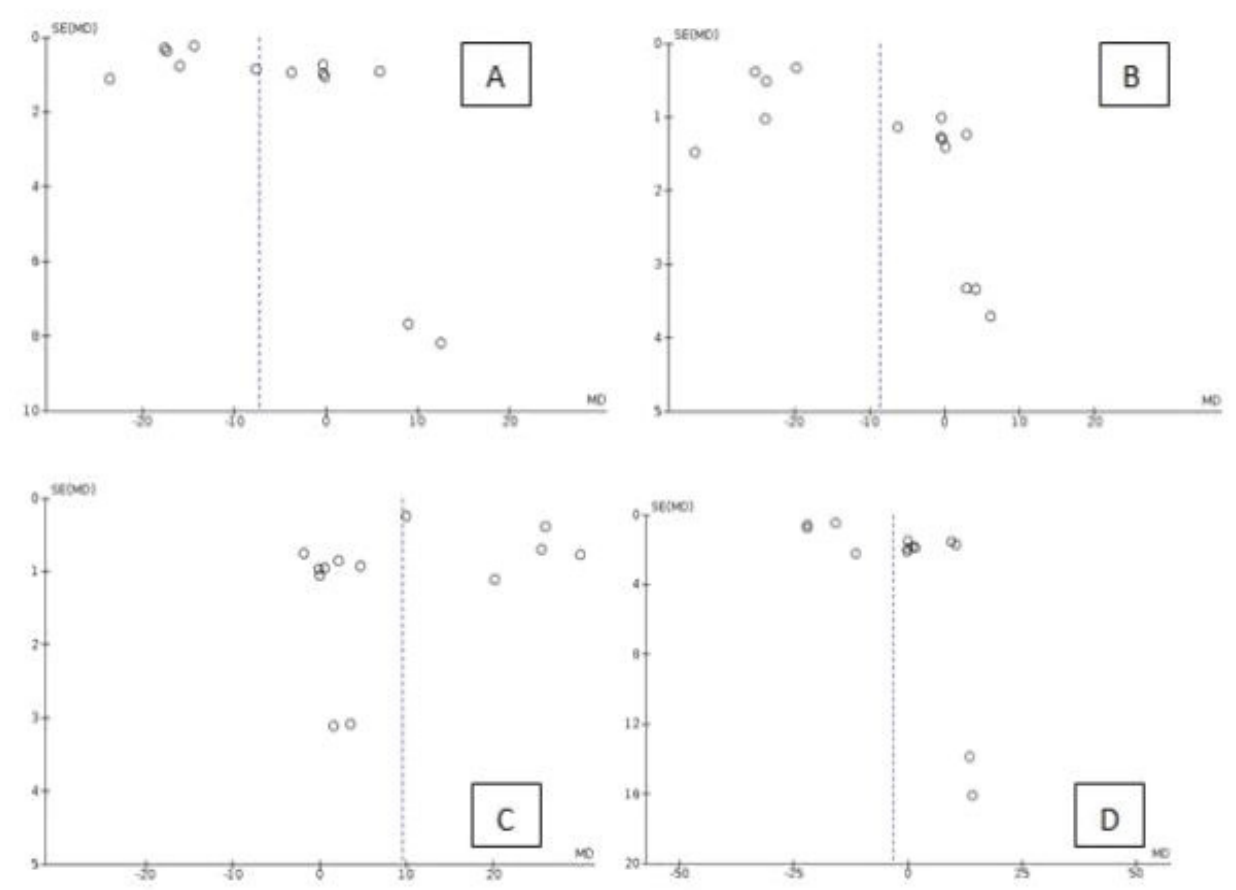

Figure 5: Funnel plots.

Subgroup analysis using 10mg of policosanol versus placebo for 12 weeks showed a total of 413 participants given policosanol while 414 were given placebo. A statistically significant result $(\mathrm{p}<0.00001)$ was found favoring policosanol in reducing total cholesterol $(-17.93[-19.85,-16.00])$, LDL (-22.08 [-25.91, -18.26]) and increasing HDL $(23.05[20.28,25.82])$. No significant results (p = 0.09) were found in terms of TAG levels. Again, the studies were highly heterogenous $(\mathrm{p}<0.00001)$ (see figures below). 
Citation: Patrocinio MP, Paragas N, Perez JA, Que DLS, Quiogue K, et al. (2017) The Effects of Sugar Cane Policosanol on the LDL, HDL, Triglyceride and Total Cholesterol Levels of Dyslipidemic Patients: A Meta-Analysis. Anesth Med Pract J: AMPJ-117. DOI: 10.29011/AMPJ-117. 100017

\begin{tabular}{|c|c|c|c|c|c|c|c|c|c|c|}
\hline \multirow[b]{2}{*}{ Study or Subgroup } & \multicolumn{3}{|c|}{ Experimental } & \multicolumn{3}{|c|}{ Control } & \multirow{2}{*}{\multicolumn{3}{|c|}{$\begin{array}{l}\text { Mean Difference } \\
\text { IV, Random, } 95 \% \mathrm{Cl}\end{array}$}} & \multirow{2}{*}{$\begin{array}{l}\text { Mean Difference } \\
\text { IV, Random, } 95 \% \mathrm{Cl}\end{array}$} \\
\hline & Mean & SD & Total & Mean 5 & SD & Total & & & & \\
\hline Berthold 10mg 2006 & 11 & 23 & 28 & -20 & 34 & 29 & $1.5 \%$ & $9,00[-6,02,24,02]$ & & \\
\hline Castano $2000^{\circ}$ & .16 .8 & 3 & 122 & 0.5 & 3 & 122 & $26.8 \%$ & $\cdot 17,30[-18,05,-16,55]$ & & 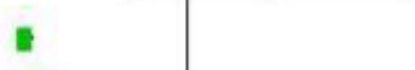 \\
\hline IInait 2013 & $.14,8$ & 3 & 30 & 1.1 & 3 & 30 & $23,8 \%$ & $\cdot 15,90[-17,42, \cdot 14,38]$ & & + \\
\hline Mas 1999 & $.17,4$ & 3 & 219 & 0.2 & 3 & 218 & $27.3 \%$ & $\cdot 17,60[-18,16,-17,04]$ & & 1 \\
\hline Torres 1995 & $.17,5$ & 3 & 14 & 6.07 & 3 & 15 & $20.5 \%$ & $-23.57[-25,76,-21,38]$ & + & \\
\hline Total (95\% Cl) & & & 413 & & & 414 & $100.0 \%$ & $.17 .93[\cdot 19.85, \cdot 16.00]$ & & $\boldsymbol{\gamma}$ \\
\hline $\begin{array}{l}\text { Heterogeneity; Tau² } \\
\text { Test for overall effect: }\end{array}$ & $\begin{array}{l}3.45 ; \mathrm{Chi} \\
Z=18.2\end{array}$ & & $\begin{array}{l}13, d f= \\
0.00001\end{array}$ & $\begin{array}{l}=4(P<) \\
1)\end{array}$ & & $001\} ;$ & $=91 \%$ & & & 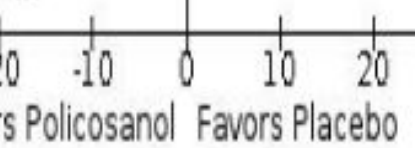 \\
\hline
\end{tabular}

Figure 6: Policosanol, 10mg vs. Placebo, 12 weeks - effect on Total cholesterol.

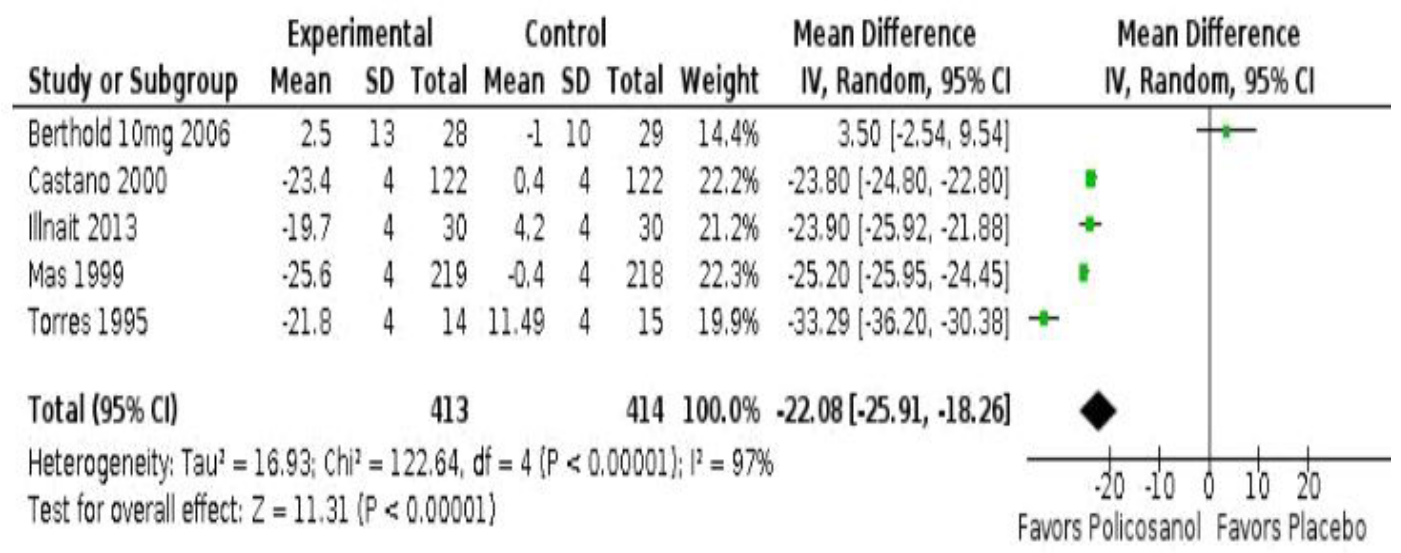

Figure 7: Policosanol, 10mg vs. Placebo, 12 weeks - effect on LDL. 
Citation: Patrocinio MP, Paragas N, Perez JA, Que DLS, Quiogue K, et al. (2017) The Effects of Sugar Cane Policosanol on the LDL, HDL, Triglyceride and Total Cholesterol Levels of Dyslipidemic Patients: A Meta-Analysis. Anesth Med Pract J: AMPJ-117. DOI: 10.29011/AMPJ-117. 100017

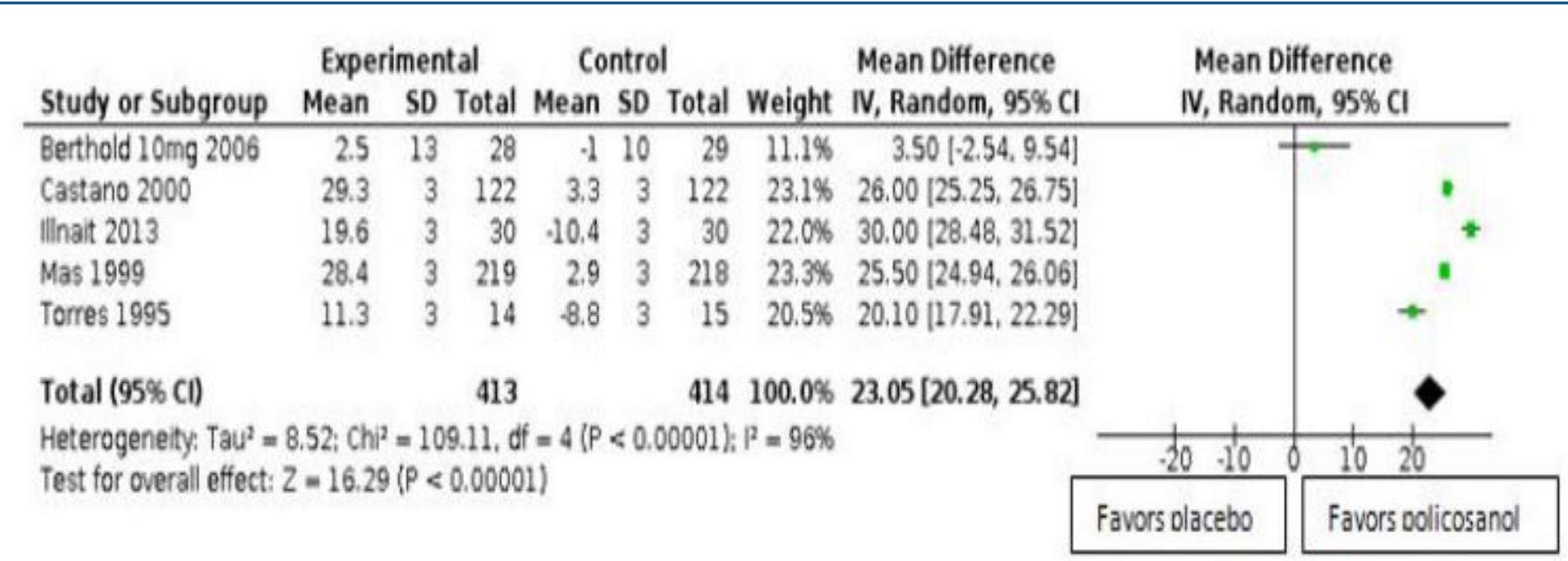

Figure 8: Policosanol, 10mg vs. Placebo, 12 weeks - effect on HDL.

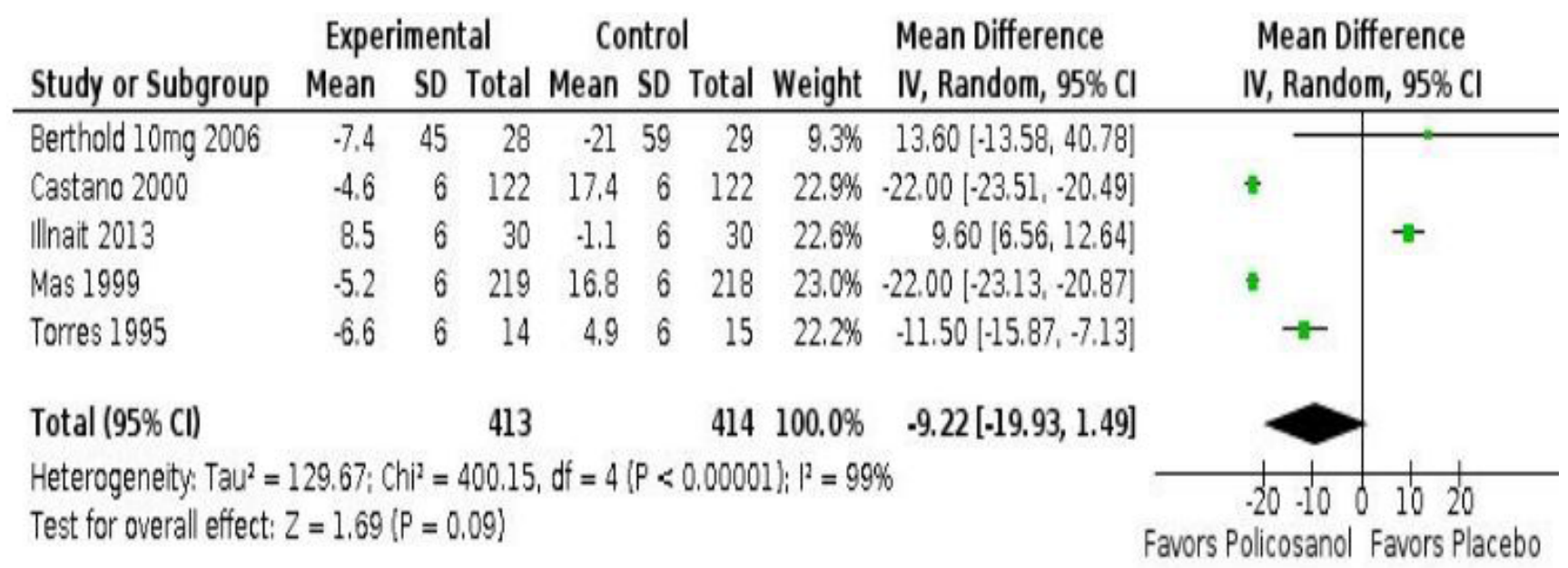

Figure 9: Policosanol, 10mg vs. Placebo, 8 weeks - effect on TAGs.

Subgroup analysis of studies that used 20mg of policosanol versus placebo for 8 weeks showed a total of 102 participants: 50 in the policosanol group and 52 in the placebo group. The confidence intervals were very wide and crossing the line of no effect. No statistically significant results in TC $(p=0.25)$, LDL $(p=0.62)$, HDL $(p=0.57)$ and TAGs $(p=0.61)$ were seen with policosanol $20 \mathrm{mg}$ for 8 weeks. There was significant heterogeneity for TC $(p=0.005, I 2=87 \%)$ and HDL $(p=0.05, I 2=74 \%)$ but not for LDL and TAGs (See Figures below). 
Citation: Patrocinio MP, Paragas N, Perez JA, Que DLS, Quiogue K, et al. (2017) The Effects of Sugar Cane Policosanol on the LDL, HDL, Triglyceride and Total Cholesterol Levels of Dyslipidemic Patients: A Meta-Analysis. Anesth Med Pract J: AMPJ-117. DOI: 10.29011/AMPJ-117. 100017

\section{Experimental Control Mean Difference Mean Difference}

\section{Study or Subgroup Mean SD Total Mean SD Total Weight IV, Random, 95\% Cl IV, Random, 95\% Cl}

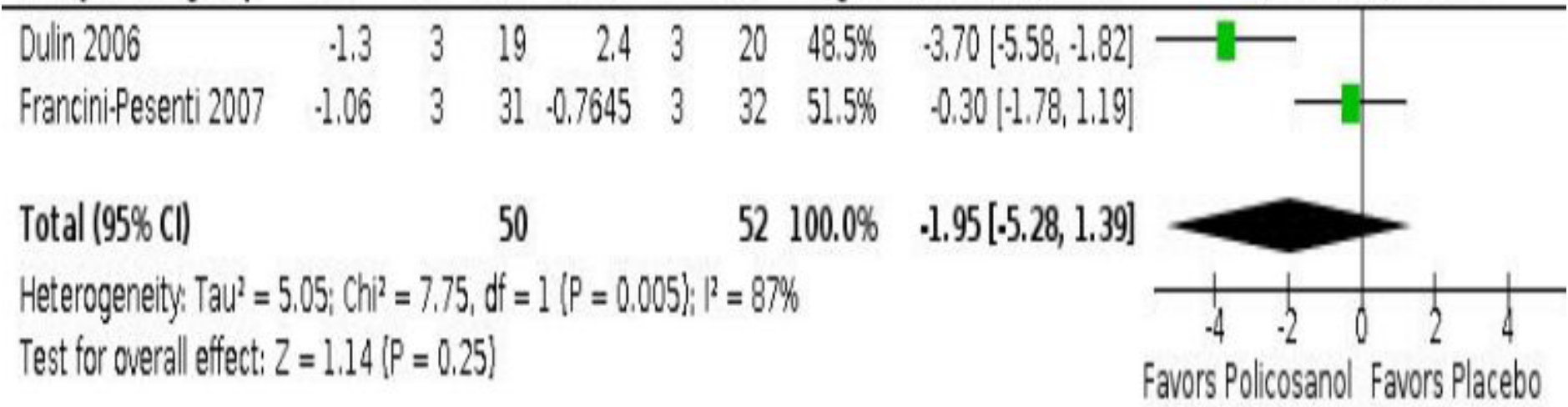

Figure 10: Policosanol, 20mg vs. Placebo, 8 weeks - effect on Total cholesterol.

\begin{tabular}{|c|c|c|c|c|c|c|c|}
\hline \multirow[b]{2}{*}{ Study or Subgroup } & \multicolumn{2}{|c|}{ Experimental } & \multicolumn{2}{|c|}{ Control } & \multicolumn{2}{|r|}{ Mean Difference } & \multirow{2}{*}{$\begin{array}{l}\text { Mean Difference } \\
\text { IV, Random, } 95 \% \mathrm{Cl}\end{array}$} \\
\hline & Mean & SD Tota & al Mean S & SD Total & Weight & IV, Random, $95 \% \mathrm{Cl}$ & \\
\hline Oulin 2006 & .7 .7 & 419 & $19 \quad .7,2$ & $4 \quad 20$ & $38.2 \%$ & $0,50|\cdot 3,01,2,01|$ & \\
\hline FrancinifFesenti 2007 & .0 .5048 & 4 & $31 \cdot 0,1694$ & 32 & $61.8 \%$ & $0.34|-2,31,1,64|$ & \\
\hline Total $(95 \% \mathrm{Cl})$ & & 50 & 50 & 52 & $100.0 \%$ & $\cdot 0.40[\cdot 1.95,1.15]$ & \\
\hline $\begin{array}{l}\text { Heterocgenety: Tau }{ }^{2}= \\
\text { Test for overall effect: }\end{array}$ & $\begin{array}{l}0.00 \mathrm{Ch}^{2}= \\
\mathrm{z}=0.50 \mathrm{ip}\end{array}$ & $\begin{array}{l}0.01, \mathrm{df}= \\
=0.621\end{array}$ & $=1(P=0.92)$ & & & & $\begin{array}{ll}2 & 2 \\
\text { Policosanol Favors P }\end{array}$ \\
\hline
\end{tabular}

Figure 11: Policosanol, 20mg vs. Placebo, 8 weeks - effect on LDL.

\begin{tabular}{|c|c|c|c|c|c|c|c|}
\hline \multirow[b]{2}{*}{ Study or Subgroup } & \multicolumn{2}{|c|}{ Experimental } & \multicolumn{2}{|c|}{ Control } & \multirow{2}{*}{\multicolumn{2}{|c|}{$\begin{array}{c}\text { Mean Difference } \\
\text { Weight IV, Random, } 95 \% \mathrm{Cl}\end{array}$}} & \multirow{2}{*}{$\begin{array}{l}\text { Mean Difference } \\
\text { IV, Random, } 95 \% \mathrm{Cl}\end{array}$} \\
\hline & Mean & SD Total & Mean SD & Total & & & \\
\hline Qulin 2006 & $.3,3$ & 3 & 393 & 20 & $46.9 \%$ & $0,60[-1,28,2,48]$ & \\
\hline Franciniffesenti 2007 & 3.2149 & 3 & 500063 & 32 & $53.1 \%$ & $=1.79(-3,28,0.31)$ & \\
\hline Total (95\% Cl) & & 50 & & 52 & $100,0 \%$ & $=0.67[-3.01,1,67]$ & \\
\hline $\begin{array}{l}\text { Heterogeneity, Tau }{ }^{2}= \\
\text { Test for werall effect: }\end{array}$ & $\begin{array}{l}2,12 ;\left(\mathrm{Chi}^{3}\right. \\
\mathrm{z}=0.56 !\end{array}$ & $\begin{array}{l}=3.83, d f= \\
=0.57]\end{array}$ & $1(P=0.05)_{i}$ & $P^{2}=747$ & & & $\begin{array}{lll}2 & 1 & 0 \\
\text { lors Placebo Fav }\end{array}$ \\
\hline
\end{tabular}

Figure 12: Policosanol, 20mg vs. Placebo, 8 weeks - effect on HDL. 
Citation: Patrocinio MP, Paragas N, Perez JA, Que DLS, Quiogue K, et al. (2017) The Effects of Sugar Cane Policosanol on the LDL, HDL, Triglyceride and Total Cholesterol Levels of Dyslipidemic Patients: A Meta-Analysis. Anesth Med Pract J: AMPJ-117. DOI: 10.29011/AMPJ-117. 100017

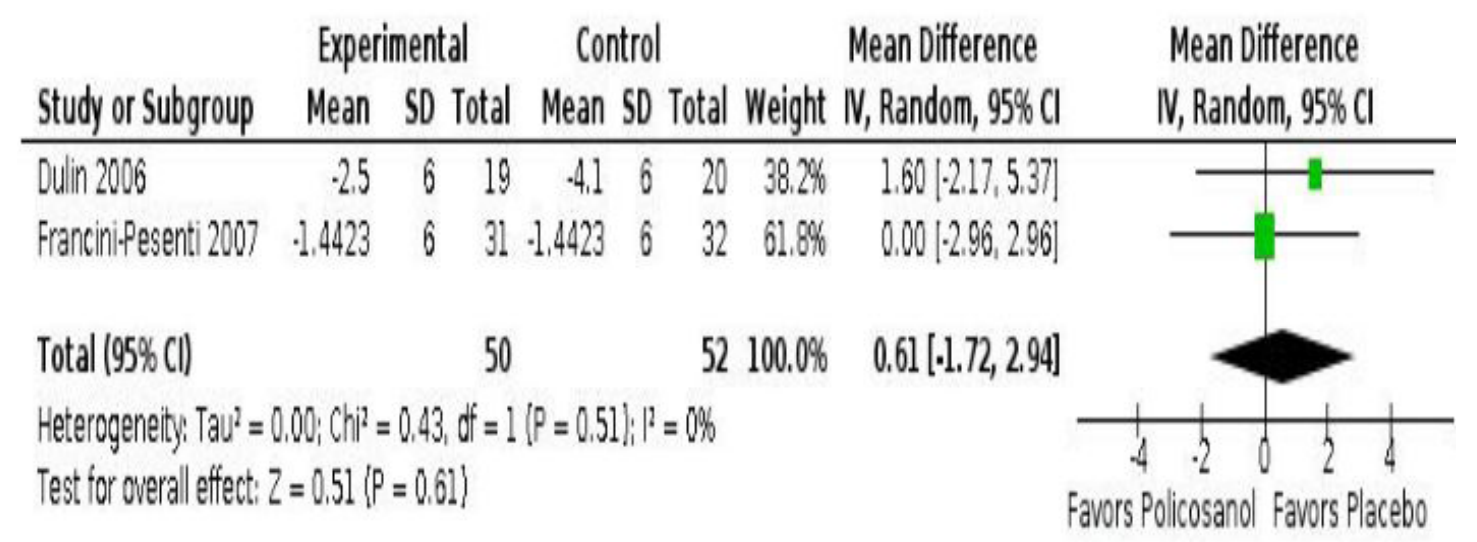

Figure 13: Policosanol, 20mg vs. Placebo, 8 weeks - effect on triglycerides.

On the other hand, subgroup analysis of studies that used $20 \mathrm{mg}$ of policosanol versus placebo for 12 weeks consisted of 87 participants who received policosanol and 88 participants who received placebo. The confidence intervals were very wide and crossed the line of no effect. No statistically significant results were found in all outcomes. Significant heterogeneity $(p<0.00001)$ was present in all except for HDL (See Figures below).

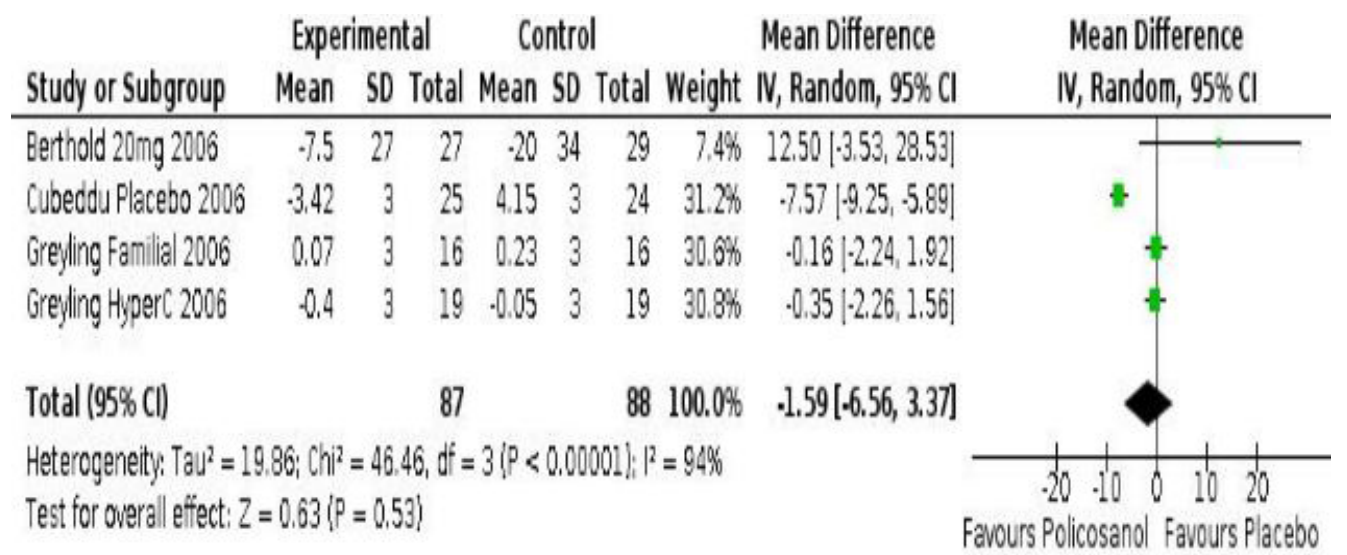

Figure 14: Policosanol, 20mg vs. Placebo, 12 weeks - effect on Total cholesterol

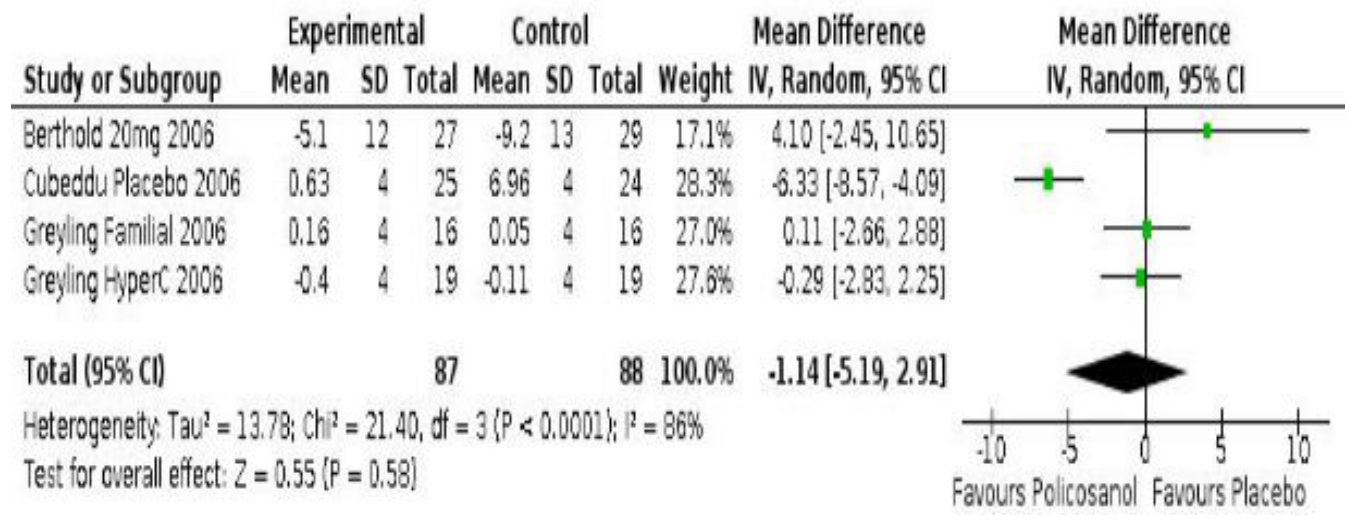

Figure 15: Policosanol, 20mg vs. Placebo, 12 weeks - effect on LDL. 
Citation: Patrocinio MP, Paragas N, Perez JA, Que DLS, Quiogue K, et al. (2017) The Effects of Sugar Cane Policosanol on the LDL, HDL, Triglyceride and Total Cholesterol Levels of Dyslipidemic Patients: A Meta-Analysis. Anesth Med Pract J: AMPJ-117. DOI: 10.29011/AMPJ-117. 100017

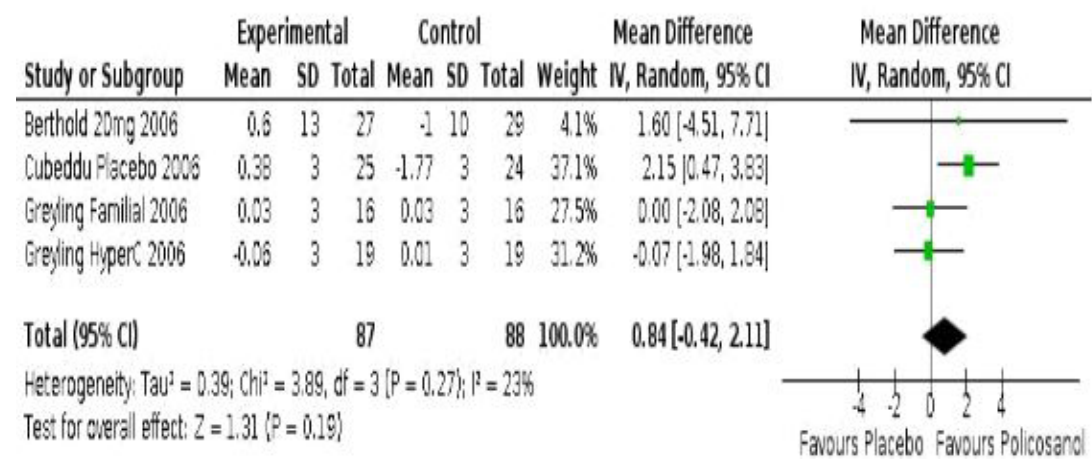

Figure 16: Policosanol, 20mg vs. Placebo, 12 weeks - effect on HDL.

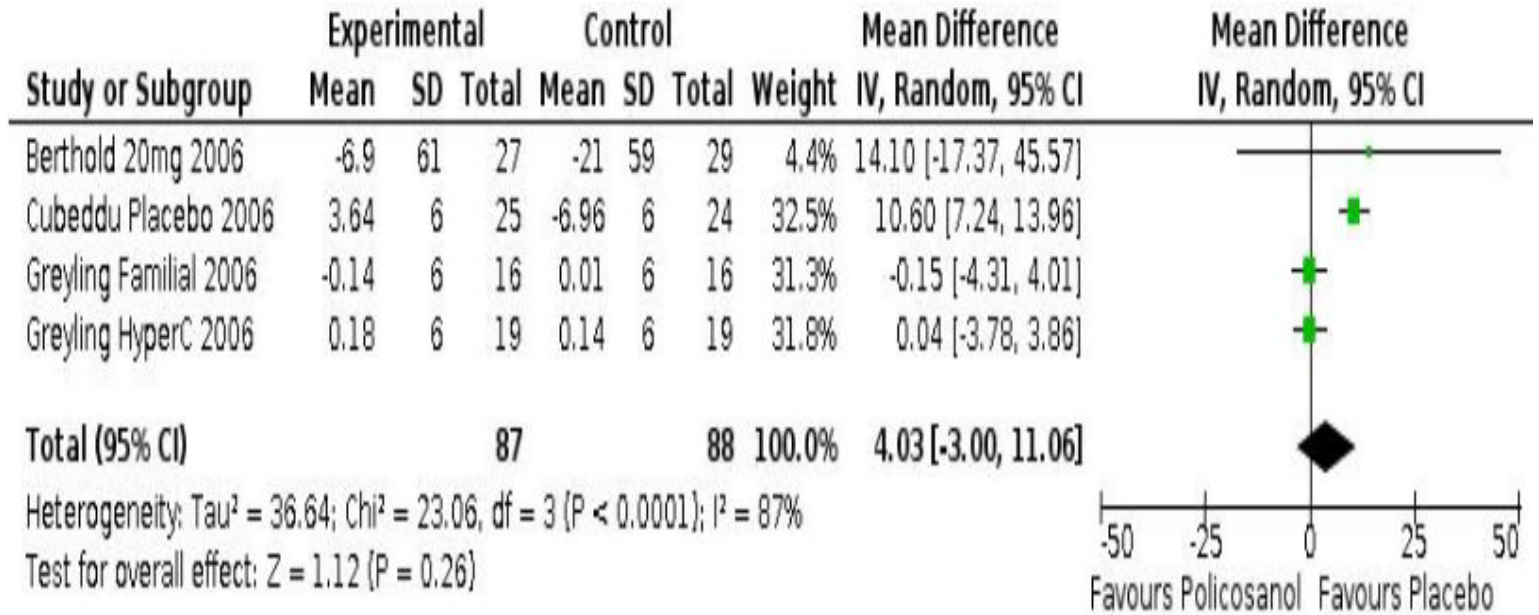

Figure 17: Policosanol, 20mg vs. Placebo, 12 weeks - effect on TAGs.

Lastly, a subgroup analysis of studies that included only the elderly and postmenopausal women 40 to 80 years of age was done. A total of 516 subjects received policosanol of variable dosage and duration, and 519 received placebo. Significant heterogeneity ( $p<$ $0.00001)$ was still found among studies. Results showed that there were significant effects for total cholesterol (-14.96 [-18.53, -11.39]), LDL (-20.23 [-26.16, -14.30]) and HDL (17.53 [7.53, 27.16]) favoring policosanol, but no significant effects were found in TAGs (See Figures below).

\begin{tabular}{|c|c|c|c|c|c|c|c|c|c|c|c|}
\hline \multirow{3}{*}{$\begin{array}{l}\text { Study or Subgroup } \\
\text { Castano } 2000\end{array}$} & \multicolumn{3}{|c|}{ Experimental } & \multicolumn{3}{|c|}{ Control } & \multirow{2}{*}{\multicolumn{2}{|c|}{$\begin{array}{l}\text { Mean Difference } \\
\text { IV, Random, 95\% CI }\end{array}$}} & \multirow{2}{*}{\multicolumn{3}{|c|}{$\begin{array}{l}\text { Mean Difference } \\
\text { IV, Random, } 95 \% \mathrm{C}\end{array}$}} \\
\hline & Mean & SD T & Total & Mean & SD T & Total & & & & & \\
\hline & .16 .8 & 3 & 122 & 0.5 & 3 & 122 & $20.5 \%$ & $-17,30[-18.05,-16.55]$ & & 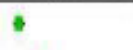 & \\
\hline Castano 2002 & -15.4 & 3 & 293 & -1 & 3 & 293 & $20.6 \%$ & $-14,40[-14,89,-13,91]$ & & - & \\
\hline Dulin 2006 & -1.3 & 3 & 19 & 2.4 & 3 & 20 & $19.6 \%$ & $-3.70 \mid-5.58,-1.82]$ & & + & \\
\hline IInait 2013 & .14 .8 & 3 & 30 & 1.1 & 3 & 30 & $20.0 \%$ & $-15,90[-17,42,-14,38]$ & & + & \\
\hline Torres 1995 & .17 .5 & 3 & 14 & 6.07 & 3 & 15 & $19.2 \%$ & $-23.57[-25.76,-21.38]$ & + & & \\
\hline Total (95\% CI) & & & 478 & & & 480 & $100.0 \%$ & $-14.96[-18.53,-11.39]$ & & & \\
\hline $\begin{array}{l}\text { Heterogeneity: Tau² = } \\
\text { Test for overall effect: }\end{array}$ & $\begin{array}{l}6.01 ; \mathrm{Chi} \\
=8.21 !\end{array}$ & & $\begin{array}{l}0.25, \mathrm{df} \\
00001\}\end{array}$ & $d f=4(P$. & $P<0.00$ & $00001\}_{i}$ & $i_{1} 1^{2}=98 \%$ & & & $\begin{array}{ll}10 & -10 \\
s \text { Policosanol }\end{array}$ & $0 \quad 10$ \\
\hline
\end{tabular}

Figure 18: Policosanol vs. Placebo in 40 to 80 years old-Effects on total cholesterol. 
Citation: Patrocinio MP, Paragas N, Perez JA, Que DLS, Quiogue K, et al. (2017) The Effects of Sugar Cane Policosanol on the LDL, HDL, Triglyceride and Total Cholesterol Levels of Dyslipidemic Patients: A Meta-Analysis. Anesth Med Pract J: AMPJ-117. DOI: 10.29011/AMPJ-117. 100017

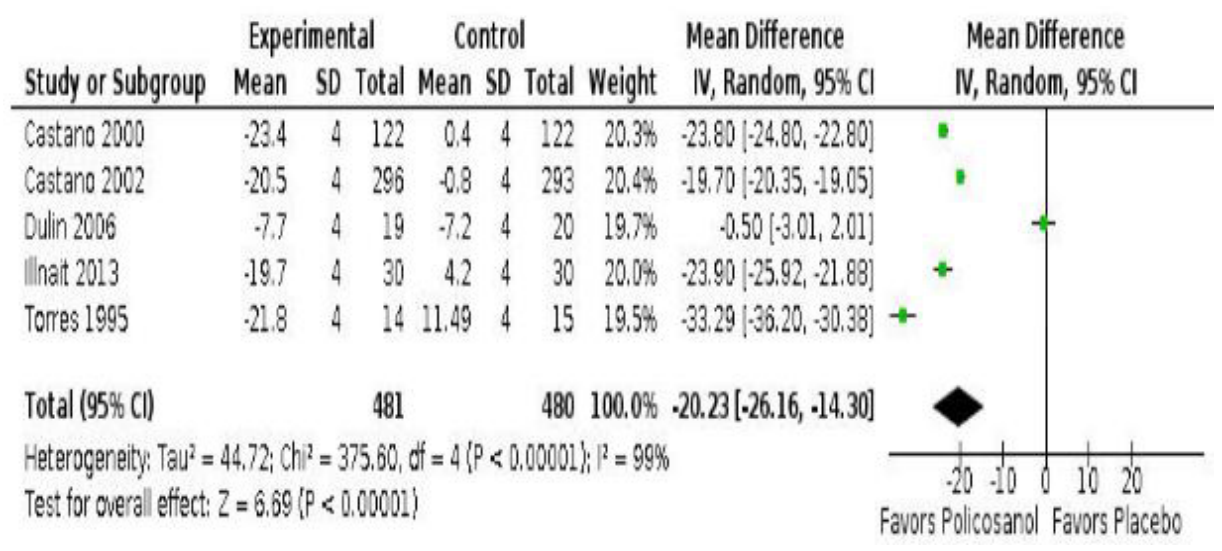

Figure 19: Policosanol vs. Placebo in 40 to 80 years old-Effects on LDL.

\begin{tabular}{|c|c|c|c|c|c|c|c|c|}
\hline \multirow[b]{2}{*}{ Study or Subgroup } & \multicolumn{2}{|c|}{ Experimental } & \multicolumn{2}{|c|}{ Control } & & \multirow{2}{*}{$\begin{array}{l}\text { Mean Difference } \\
\text { IV, Random, } 95 \% \mathrm{CI}\end{array}$} & \multirow{2}{*}{$\begin{array}{l}\text { Mean Difference } \\
\mathrm{N} \text {, Random, } 95 \% \mathrm{Cl}\end{array}$} & \\
\hline & Mean & SD Total & Mean SI & id Total & Weight & & & \\
\hline Castano 2000 & 29.3 & 3122 & 3.3 & $3 \quad 122$ & $20.1 \%$ & $26.00\{25.25,26.75)$ & & + \\
\hline Castano 2002 & 127 & 296 & 2.7 & 3293 & $20.1 \%$ & $10,00[9.52,10,48]$ & ' & \\
\hline Dulin 2006 & .3 .3 & 19 & .3 .9 & 20 & $19.9 \%$ & $0.60|-1.28,2.48|$ & + & \\
\hline Ilnait: 2013 & 19.6 & 30 & $\cdot 10,4$ & 3 & $20.0 \%$ & $30.00|28,48,31.52|$ & & $*$ \\
\hline Torres 1995 & 11.3 & 14 & -8.8 & 15 & 19.98 & $20.10[17.91,22.29]$ & & + \\
\hline Total (95\% Cl) & & 481 & & 480 & $100.0 \%$ & $17.35[7.53,27.16]$ & & \\
\hline $\begin{array}{l}\text { Heterogeneity: Tau' = } \\
\text { Test for overall effect: }\end{array}$ & $\begin{array}{l}24,84 ; 0 \\
=3,46\end{array}$ & $\begin{array}{l}i^{2}=1848,08 \\
P=0,0005]\end{array}$ & $8 \mathrm{Cl}=4 \mathrm{LF}$ & $P<0,000$ & $01] ;\left.\right|^{1}=1$ & $00 \%$ & $\begin{array}{cccc}20 & 10 & 10 & 10 \\
\text { Favors Placebo Favors }\end{array}$ & icosa \\
\hline
\end{tabular}

Figure 20: Policosanol vs. Placebo in 40 to 80 years old-Effects on TAGs.

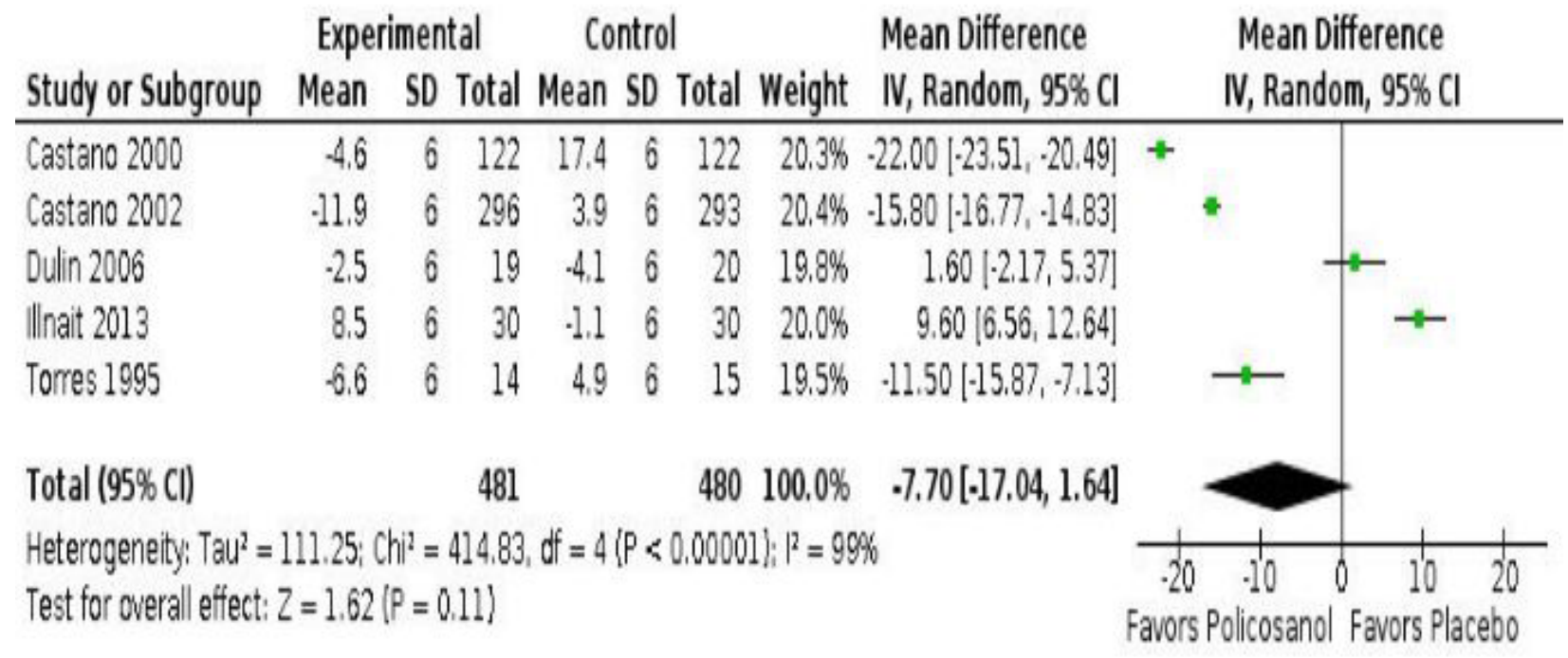

Figure 21: Policosanol vs. Placebo in 40 to 80 years old-Effects on TAGs. 


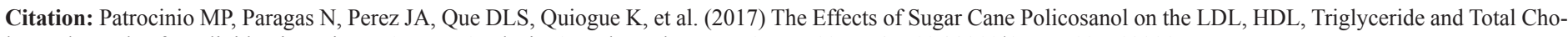
lesterol Levels of Dyslipidemic Patients: A Meta-Analysis. Anesth Med Pract J: AMPJ-117. DOI: 10.29011/AMPJ-117. 100017

Aside from the lipid levels, majority of the studies also looked into the adverse effects of policosanol on the subjects. No adverse effects were reported in the studies by Torres (1995) [5]. Dulin (2006) [12] reported that policosanol was well tolerated with similar side effect profile to placebo. Illnait (2013) [8] likewise reported that policosanol was safe and well-tolerated; some of the minimal adverse events reported included dizziness and vertigo, somnolence, and heartburn but these consisted of less than $5 \%$ of those given policosanol. Gastrointestinal symptoms were reported in two cases out of 31 subjects (Francini-Pesenti, 2007) [15] and one out of 219 subjects (Mas, 1999) [6] who received policosanol therapy. In the study by Berthold (2006), 45 patients were reported to have at least one adverse effect after treatment, these included bronchitis $(13 \%)$, back pain $(11 \%)$, pharyngitis $(9 \%)$, URTI $(7 \%)$, and gastritis (5\%). Two cases of headache and one case of vertigo were reported during intake of policosanol in the study of Greyling in 2006 , these accounted for less than $1 \%$ of the population.

\section{Discussion}

The present meta-analysis showed that policosanol in general significantly reduced total cholesterol, LDL and HDL levels. These effects were observed in short term treatment with $10 \mathrm{mg}$ policosanol for 8 weeks. A higher dose of policosanol at $20 \mathrm{mg}$ regardless of treatment duration, however did not show such effects. The results of this study are inconsistent with the previous metaanalysis done by Ogbac, etal. (2010) [18] which demonstrated no significant difference in mean end point levels of total cholesterol and LDL among patients with hypercholesterolemia compared to placebo and thus does not recommend the use of policosanol for these patients. It should be noted that the study by Ogbac etal in 2010 [18] included only three eligible studies, which may have contributed to the absence of a significant treatment effect. In addition, all three studies included in Ogbac's meta-analysis, specifically Dulin (2006) [12] and Berthold (2006) [10] did not show any significant effect in any of the lipid profile parameters. The present meta-analysis consisted of 10 clinical trials, one of which [8] is fairly recent and has not been included in the previous meta-analysis. The results of the present meta-analysis are consistent with individual studies by Illnait in 2013 [8], Torres in 1995 [5], Castano in 2002 [16], Castano in 2000 [7] and Mas in 1999 [6] although the percent decrease in total cholesterol and LDL and percent increase in HDL were generally lower at $-7.24 \%,-8.65 \%$ and $+9.88 \%$, respectively than in previous studies at $-15.4-25.6 \%,-17.4-25.2 \%$, $+11.3-28.4 \%$, respectively. Among the previous studies $[5,8]$ had the nearest values for percent change of total cholesterol, LDL and HDL at $-17.5 \%,-19-21 \%$, and $+11.3 \%$, respectively.

A consequence of including a greater number of trials is the higher likelihood for heterogeneity because of various contributing factors within each study. The funnel plots reflected this heterogeneity among studies in terms of patient population and meth- odology. The substitution imputation could have likewise affected the funnel plot as the effects of each study were altered. Unlike the previous meta-analysis which included only subjects with LDL cholesterol levels above $130 \mathrm{mg} / \mathrm{dL}$ with no other co-morbidities, the present study also included two studies that had subjects with comorbidities like diabetes mellitus and hypertension which may have contributed to varied responses to policosanol. The present study aims to show a comprehensive analysis of the effects of policosanol in dyslipidemic individuals regardless of their comorbid conditions. Therefore, more varied studies were included despite their possibility of contributing to heterogeneity. In addition, it is common for dyslipidemic Filipino patients to have hypertension or diabetes as comorbidities. No studies have shown that hypertension or diabetes could affect the absorption, metabolism and effects of policosanol.

Subgroup analyses were performed in order to remove possible sources of heterogeneity. First, the studies were sub-grouped according to treatment parameters used-dosage of policosanol and duration of treatment the authors deemed this contributory to heterogeneity of the studies. After analyzing the studies in each subgroup, significant heterogeneity was still found.

For the subgroup of studies that used 10mg policosanol versus placebo for 12 weeks $[5-8,10]$, the mean age of subjects varied from 54 to 62 . Though there is a difference in age groups, it is not likely contributory to significant heterogeneity since the mean age is almost comparable. The studies used different components of the lipid profile tests as inclusion criteria for recruiting subjects however all except Illnait, 2013 [8] included LDL levels as criteria for subject selection. The diet plans and duration of diet program conducted by each study were also varied and therefore may be a source of heterogeneity. Some studies included a $5 \mathrm{mg} 12$ week treatment before giving the $10 \mathrm{mg} 12$-week treatment, making the duration of policosanol intake 24 weeks in total. In effect, the lipid profiles obtained were after 24 weeks of intervention. Nothing in the literature clearly states the optimal dose and duration of policosanol treatment; therefore, the present meta-analysis did not put any limits to these parameters. However, the authors decided to exclude studies that made use of more than $20 \mathrm{mg}$ of policosanol and only 4 weeks of treatment as these were considered extremes.

The significant heterogeneity observed between the two studies that used $20 \mathrm{mg}$ of policosanol for 8 weeks may be due to differences in certain demographic parameters of the subjects, specifically sex, age and body-mass index (BMI), as well as dietary practices adopted during the study. Although both studies by Dulin et al (2006) [12] and Francini-Pesenti et al (2007) [15] recruited primary hypercholesterolemic subjects, more females $(70 \%)$ were enrolled in the first, whereas an even number of males and females were enrolled in the latter. In terms of age, the mean age is younger in Dulin's study, ranging from 42-47 years, while Francini-Pesen- 


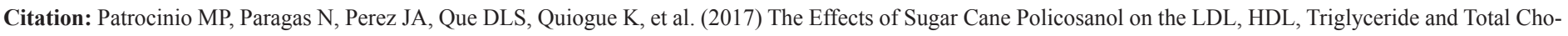
lesterol Levels of Dyslipidemic Patients: A Meta-Analysis. Anesth Med Pract J: AMPJ-117. DOI: 10.29011/AMPJ-117. 100017

ti's study had relatively older subjects, with 52-54 years of age for both policosanol and placebo groups. In terms of baseline BMI, the subjects in Dulin's study were overweight $(\mathrm{BMI}=30)$, whereas to those in Francini-Pesenti'sstudyhave normal BMI (24). Although the subjects' lifestyles were unmodified during the course of the study, those in Francini-Pesenti's trial underwent a normocaloric dietary regimen while those in Dulin's study continued their usual dietary habits, with no food restriction which may alter their serum lipid levels.

In the subgroup of studies that used $20 \mathrm{mg}$ policosanol versus placebo for 12 weeks, time of administration of the treatment may have contributed to heterogeneity. In the study by Berthold (2006) [10] and Greyling (2006) [9], policosanol was administered together with and after an evening meal, respectively. For Cubeddu 2006 there was no specific time of intake. Cholesterol levels are generally increased during the evening (Greyling, 2006) [9] probably because of the physiologic nocturnal increase in hepatic synthesis of cholesterol. Since policosanol acts by inhibiting hepatic cholesterol synthesis, it is therefore recommended to administer the medication during evening. Administering policosanol anytime within the day may not maximize its effects. Also in this subgroup, Greyling (2006) [9] specifically separated subjects who had familial hypercholesterolemia and these patients generally had higher levels of LDL at about $200 \mathrm{mg} / \mathrm{dL}$ compared to those of the subjects in the other studies (ranging from 140 to $167 \mathrm{mg} / \mathrm{dL}$ ). Additionally, only patients from the study of Berthold included smokers among their study population. The distinction between familial and non-familial hypercholesterolemia and smoking may have been enough to produce significant heterogeneity in between the studies. It should also be noted that Greyling (2006) [9] used a crossover method however the authors of the present study believe that this would not have significantly contributed to the heterogeneity as even if the baseline would be altered by the switching of treatments, what would be measured is the percent change after treatment.

The studies included in the present meta-analysis had subjects with age ranging from 18 to 80 years old. With this wide age range, there is a strong possibility that cholesterol levels of the subjects significantly varied and may have affected response to treatment. Particularly in postmenopausal women in which there is often a rapid rise in cholesterol levels. To minimize the effect of widely varied age in the results of the meta-analysis, the authors decided to add a subgroup for studies that included subjects who were 40 years old and above. Results remained to be the same as prior to subgrouping and heterogeneity was still present. Some differences between the studies included in this subgroup that may have accounted for the significant heterogeneity include the duration of follow-up and the type of subjects themselves. The duration of follow-up was different for the six studies: one was eight weeks [12]; three studies used 12 weeks $[5,8,16]$; and the study by Casta- no et al. (2002) [16] had 12 months as the duration for follow-up. Castano et al. (2000) [16] also used postmenopausal women only who still had elevated levels of TC and LDL despite adherence to a 6- week cholesterol diet. This may mean that these subjects are diet resistant and may benefit more with intake of medications.

Policosanol was shown to be well-tolerated and relatively safe. Side effects were very minimal and occurred only in less than $1-5 \%$ of the study population who received policosanol treatment. Among the side effects noted, gastrointestinal symptoms were the most consistent followed by neurological symptoms including headache, dizziness and vertigo.

A major strength of this present meta-analysis is that it provided a thorough and comprehensive background on the treatment effect of policosanol regardless of age. It has put together and analyzed altogether the results of available and related literature regarding the possible benefits of policosanol. Limitations of this study are that the studies included were heterogenous. The heterogeneity of studies even after subgroup analysis may show that the available literature regarding the use of policosanol is still very diverse specifically in terms of subjects, treatment dosage and duration and timing of administration of treatment. Sensitivity analysis was also attempted to achieve homogeniety, but the artificially magnified weights of each study made it difficult to achieve reliable results. When studies were removed, homogeniety was achieved, but at the cost of validity. The remaining studies were very similar to each other and it is the researchers' opinion that by the time apparent homogenousity was achieved, the meta-analysis is at high risk of selection bias and the file drawer effect.

Majority of the standard deviations used in this meta-analysis were imputed and it is possible that the process artificially magnified the studies' heterogeneity. The standard deviations used were relatively small and this could have increased the weights of each study. Therefore, the effects of studies with opposing results were great, leading to artificial heterogeneity. The researchers defend their decision to impute on 2 bases. First, imputation by substitution was performed rather than by the correlation coefficient method because the latter produced even more precise standard deviations. The resultant standard deviations ranged from 0.1 to 0.9 . Inspection of the results showed unrealistically precise and consistent data. Furthermore, the correlation coefficient method reduced the weight of the studies that reported actual standard deviations to almost $0 \%$. At an intuitive level, this method was gravely erroneus. It was the researchers' opinion that substitution was a better alternative and using correlation coefficients would have severely affected the current study's validity. Second, all authors reported standard deviations for the raw values of their studies' lipid panels, but not all reported standard deviations in their percent change. Despite this, the researchers persisted in using percent change as the current study's primary outcome because it is a more useful 


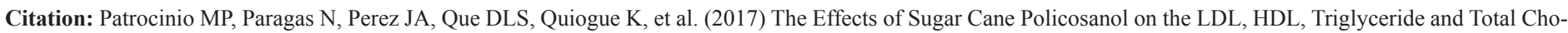
lesterol Levels of Dyslipidemic Patients: A Meta-Analysis. Anesth Med Pract J: AMPJ-117. DOI: 10.29011/AMPJ-117. 100017

laboratory measure than the absolute values of the lipid panel. In the clinical setting, changes in lipid profile are more meaningful than their raw values when evaluating a therapeutic intervention.

Nevertheless, the results of this study showed that policosanol does have some benefit in lowering cholesterol levels and increasing HDL in patients with hypercholesterolemia regardless of age, with very minimal and insignificant side effects. Its use may therefore be recommended. However, more studies are needed to establish the actual dose of policosanol and duration of treatment considered most optimal and most efficient. Future studies exploring the effects of policosanol in lipid profile functions of particularly elderly people aged 60 years old and above are also recommended in order to lessen the variability of treatment effect brought about by age. Another option may be being to stratify the subjects within the study by age in order to compare if there are actual differences in response between subjects of different age groups.

\section{Conclusion}

Policosanol was found to significantly improve the lipid profile parameters specifically total cholesterol, LDL-C and HDL-C but not TAGs in dyslipidemic patients at a dose of $10 \mathrm{mg}$ for 8 weeks. This was contradictory to the results of previous metanalysis. No significant effect was seen with policosanol dose to $20 \mathrm{mg}$. The heterogenieity problems encountered in this study may reflect the inconsistent and highly variable state of current literature and may be attributed to differences in methodologies and demographic parameters of the subjects. There is a great need to standardize important variables such as the patient population, intervention, controls and the outcome measures. No significant side effects were noted with policosanol use and hence its use may be considered as safe and well tolerated.

\section{References}

1. WHO (2014) Country Profile for Non-Communicable Diseases.

2. Marinangeli CP, Kassis AN, Jain D, Ebine N, Cunnane SC, et al. (2007) Comparison of composition and absorption of sugarcane policosanols (abstract) Br J Nutr 97: 381-388.

3. Singh DK, Li L, Porter TD (2006) Policosanol inhibits cholesterol synthesis in hepatoma cells by activation of AMP-kinase". J Pharmacol Exp Ther 318: 1020-1026.

4. Liu S, Tan MY, Zhao SP, Rong H (2012) Effects of policosanol on serum lipids and heme oxygenase-1 in patients with hyperlipidemia". Zhonghua xin xue guan bing za zhi 40: 840-843.

5. Torres O, Agramonte AJ, Illnait J, Más Ferreiro R, Fernández L (1995) Treatment of hypercholesterolemia in NIDDM with policosanol. Diabetes Care 18: 393-397.
6. Más R, Castaño G, Illnait J, Fernández L, Fernández J (1999) Effects of policosanol in patients with type II hypercholesterolemia and additional coronary risk factors. Clinical Pharmacology and Therapeutics 65: 439-447.

7. Castaño G, Más R, Fernández L, Fernández JC, Illnait J (2000) Effects of Policosanol on Postmenopausal Women with Type II Hypercholesterolemia. Gynecol Endocrinol 14: 187-195.

8. Illnait J (2013) Effects of policosanol (5 and 10mg/day) in adults with serum cholesterol levels $<=5.9 \mathrm{mmol} / \mathrm{L}$. International Journal of Pharmacological Science Rev Res 22: 303-309.

9. Greyling A, De Witt C, Oosthuizen W, Jerling JC (2005) Effects of a policosanol supplement on serum lipid concentrations in hypercholesterolaemic and heterozygous familial hypercholesterolaemic subjects. British Journal of Nutrition 95: 968-975.

10. Berthold HK, Unverdorben S, Degenhardt R, Bulitta M, Gouni-Berthold I (2006) Effect of Policosanol on Lipid Levels among Patients with Hypercholesterolemia or Combined Hyperlipidemia: A Randomized Controlled Trial JAMA 295: 2262-2269.

11. Cubeddu LX, Cubeddu RJ, Heimowitz T, Restrepo B, Lamas GA (2006) Comparative Lipid Lowering Effects of Policosanol and Atorvastatin: A Randomized, Parallel, Double-Blind, Placebo-Controlled Trial. Am Heart J 152: 982.e1-5.

12. Dulin MF, Hatcher LF, Sasser HC, Barringer TA (2006) Policosanol in Ineffective in the Treatment of Hypercholesterolemia: A Randomized Controlled Trial. Am J Clin Nutr 84: 1543-1548.

13. Kassis AN, Jones PJ (2006) Lack of Cholesterol-Lowering Efficacy of Cuban Sugar Cane Policosanols in Hypercholesterolemia Persons. American Journal of Clinical Nutrition 84: 1003-1008.

14. Kassis AN, Kubow S, Jones PJ (2009) Sugar Cane Policosanol do not reduce LDL Oxidation in Hypercholesterolemic Individuals. Lipids 22: 391-396.

15. Francini-Pesenti F, Beltramolli D, Dall'acqua S, Brocadello F (2008) Effect of Sugar Cane Policosanol on Lipid Profile in Primary Hypercholesterolemia. Phytother Res 22: 318-322.

16. Castaño G, Mas R, Fernández L, Illnait J, Mesa M (2002) Comparison of the Efficacy and Tolerability of Policosanol with Atorvastatin in elderly patients with Type II Hypercholesterolemia. Drugs Aging. 20: 153-163.

17. Chen JT, Wesley R, Shamburek RD, Pucino F, Csako G (2005) Meta-analysis of natural therapies for hyperlipidemia: Plant sterols and stanols versus policosanol. Pharmacotherapy 25: 171-183.

18. Ogbac F, Quimpo R, Hilado JE, Sy RA, Luz V, et al. (2010) A MetaAnalysis on Sugar Cane Policosanol as Treatment for Hypercholesterolemia. Philippine Journal of Internal Medicine 48: 26-32.

19. The Clinical Practice Guidelines for the Management of Dyslipidemia in the Philippines. 